\title{
Transformation of myelodysplastic syndrome to acute megakaryoblastic leukemia: An aggressive disease
}

\author{
Ganesh Kasinathan ${ }^{1}$ and Bee Sun Lee ${ }^{2}$ \\ ${ }^{1}$ Ampang Hospital \\ ${ }^{2}$ Ampang Hospital.
}

November 17, 2020

\begin{abstract}
A 59-year old gentleman with transformation of myelodysplastic syndrome to acute megakaryoblastic leukemia was treated with standard intensive chemotherapy. His poor outcome was attributed to advanced age, aggressive disease biology, underlying myelodysplastic syndrome, poor response to induction chemotherapy, high lactate dehydrogenase and lack of good cytogenetic and molecular mutations.
\end{abstract}

\section{Introduction}

Acute megakaryoblastic leukemia (AMKL) is a subtype of acute myeloid leukemia (French-American-British classification AML-M7) which is defined as $50 \%$ or more of total blast cells in a bone marrow sample being attributed to megakaryoblasts ${ }^{1}$. As with other subtypes of acute myeloid leukemia (AML), the total blasts of more than $20 \%$ of all nucleated cells in the peripheral blood film and bone marrow also applies to AMKL. AMKL was first described by Von Boros, et al in 1931. AMKL may occur as de novo, or secondary to a myelodysplastic syndrome (MDS) or myeloproliferative neoplasm (MPN). AMKL shows a bimodal age distribution; being common in children aged 1-3 and elderly adults. Down syndrome (DS) individuals have a 500-fold increase in contracting AMKL, and they have a better prognosis as compared to non-DS individuals ${ }^{2}$. There is also an association between mediastinal germ cell tumours (GCT) and AMKL in young male children with many of them being diagnosed with AMKL a few months later ${ }^{3}$. AMKL is known to be associated with extensive myelofibrosis resulting in a frequent dry bone marrow aspiration. This disease demonstrates a poor prognosis with the median survival of less than 6 months from diagnosis ${ }^{4}$. Here, we report an unusual case of transformation of myelodysplastic syndrome to acute megakaryoblastic leukemia in a middle-aged male who followed an aggressive course of illness.

\section{Case Presentation}

A 59-year old gentleman of Malay ethnicity who had no past medical history, presented to the department of hematology with fever, lethargy, headache, skin bruising, anorexia, and loss of weight for the past 3 weeks. He also complained of gingival bleeding which occurred spontaneously. He had no significant family history. He was a non-smoker and a teetotaler. He had no history of consuming recreational drugs. He works as a construction worker. He is married with 5 children, whom are all healthy.

Physical examination revealed an alert and medium-built gentleman. His Eastern Cooperative Oncology group (ECOG) performance status was 0 (fully active). He had no dysmorphic features. He was pale with a blood pressure was 110/60 and pulse rate of 96 beats per minute. He had noticeable cutaneous bruising on the lower limbs. The cardiovascular and respiratory systems were unremarkable on examination. There was no palpable lymphadenopathy or organomegaly. 
The complete blood count (CBC) which was analysed by an automated haematologic analyser (Sysmex, XE5000, Vienna, Austria) showed severe peripheral pancytopenia; haemoglobin of $6.5 \mathrm{~g} / \mathrm{dL}$, total white blood cell count of $1.5 \times 10^{9} / \mathrm{L}$ and a platelet count of $21 \times 10^{9} / \mathrm{L}$. He had an elevated lactate dehydrogenase (LDH) of $550 \mathrm{U} / \mathrm{L}$. His serology screen was negative for viral hepatitis and Human Immunodeficiency Virus (HIV). The peripheral blood film which was stained with Wright-Giemsa revealed red blood cell anisopoikilocytosis, nucleated red blood cells, numerous mononuclear cells and giant platelets. The chest radiograph did not show any mediastinal widening. The bone marrow aspiration which was stained with May-Grunwald-Giemsa (Figure 1A) showed hypercellular particles for age and trilineage dysplasia with $52 \%$ megakaryoblasts. The megakaryoblasts were described as agranular, medium-to-large cells with round nucleus, basophilic cytoplasm with cytoplasmic blebs and pseudopods. Erythropoiesis and granulopoiesis were suppressed. Bone marrow trephine biopsy (Figure 1B) showed topographical dysplasia, marrow fibrosis and infiltration by clusters of megakaryoblasts. The tumour cells (Figure 1C-E) stained positive for CD33, CD34 and CD61. They were negative for MPO. The immunohistochemical stains used in this specimen originated from Dako Artisan Link, Denmark. Immunophenotyping of the bone marrow sample by 8-colour flow cytometer (BD FACSCanto II) showed 55\% cluster of blasts expressing CD13, CD33, CD34, CD36, CD41 and CD61 and were negative for cMPO and HLA DR. Silver impregnation stain of the trephine biopsy (Figure 1F) showed increased reticulin fibrosis (World Health Organisation 2016 grading system for bone marrow fibrosis: Grade 2). Cytogenetic studies using Giemsa banding technique revealed $-7 \mathrm{q}$ and trisomy 8 abnormalities.

He was diagnosed as transformation of myelodysplastic syndrome to acute megakaryoblastic leukemia. He was induced with standard combination chemotherapy consisting of daunorubicin $\left(60 \mathrm{mg} / \mathrm{m}^{2}\right)$, an anthracycline for three days, and continuous infusion of cytarabine $\left(100 \mathrm{mg} / \mathrm{m}^{2}\right)$, an antimetabolite for seven days. However, he was refractory to induction chemotherapy. He was re-induced with FLAG chemotherapy (fludarabine $30 \mathrm{mg} / \mathrm{m}^{2}$ and high dose cytarabine $2 \mathrm{~g} / \mathrm{m}^{2}$ once daily for 5 days), in which he failed to respond. He succumbed to his illness at 7 months of diagnosis.

\section{Figure 1}

Figure 1: (A) The bone marrow aspirate demonstrates hypercellularity for age, trilineage dysplasia with $52 \%$ megakaryoblasts. The megakaryoblasts are described as agranular, medium-to-large cells with round nucleus, scanty basophilic cytoplasm with cytoplasmic blebs and pseudopods. Erythropoiesis and granulopoiesis are suppressed. (B) H \& E stain of trephine biopsy (x 40 magnification) shows infiltration of marrow by large clusters of megakaryoblasts. (C-E) A panel of immunohistochemical staining, Dako, Denmark (x 40 magnifications) shows positivity for CD34 and CD61 and negativity for MPO. (F) Reticulin stain shows Grade 2 marrow fibrosis.

\section{Discussion}

We describe a rare case of transformation of MDS to AMKL in a middle-aged male who did not respond to standard chemotherapy. Our patient presented with pancytopenia at diagnosis, which is consistent with most literature on AMKL, but in $30 \%$ of the cases, patients may present with leucocytosis ${ }^{5}$. Organomegaly in the form of hepatosplenomegaly and lymphadenopathy is commonly seen in AMKL unlike other subtypes of AML. AMKL is more frequently seen in children with an incidence of $3-10 \%$ whereas, only $1.2 \%$ of adult AML can be attributed to $\mathrm{AMKL}^{6}$.

On cytomorphology, megakaryoblasts are described as pleomorphic, medium-to-large in size which exhibit scanty cytoplasm, inconspicous nucleoli and cytoplasmic pseudopods. Megakaryoblasts show diffuse reaction on Periodic acid Schiff (PAS), non-specific esterase (NSE) and acid phosphatase cytochemistry ${ }^{7}$. On electron microscopy, megakaryoblasts express platelet peroxidase $^{8}$.

AMKL may arise as de novo, or from a pre-existing myelodysplastic syndrome (MDS) such as seen in our case, in which, the latter has a worse prognosis. Megakaryoblasts often express Factor VIII, CD13, CD33, CD34, CD36, CD38, CD41 (Gp IIb/IIIa), CD42b (Gp Ib) and CD 61(Gp IIIa) on immunophenotyping analysis $^{8}$. The tumour cells are negative for CD117, HLA DR and cMPO. 
Common molecular abnormalities encountered in AMKL are mutations of the megakaryoblastic leukemia protein-1 (MKL-1), and GATA1 gene ${ }^{9}$. The tumour cells in AMKL are thought to overexpress multidrug resistant-1 gene (MDR-1) and P-glycoprotein (P-gp) which contribute to its poor prognosis ${ }^{9}$. Cytogenetic abnormalities are more frequently encountered in AMKL than in other subtypes of AML. They are trisomy $8,-7 \mathrm{q},-5 \mathrm{q}$ and balanced reciprocal chromosomal translocations such as $\mathrm{t}(1 ; 22), \mathrm{t}(1 ; 5)$ and $\mathrm{t}(8 ; 17)^{9}$. Cytogenetic abnormality $\mathrm{t}(1 ; 22)$ is commonly associated with AMKL in children, more extensive myelofibrosis and cytopenia ${ }^{10}$. The presence of $\mathrm{t}(9 ; 22)$ abnormality may indicate the transformation of an underlying chronic myeloid leukemia (CML).

The differential diagnosis for AMKL is pure erythroid leukemia (PEL) and MDS-erythroleukemia. The World Health Organization (WHO) 2016 has reclassified PEL as the only subtype of de novo acute erythroid leukemia (AEL), and erythroleukemia into the MDS category ${ }^{11}$. PEL is defined as clonal proliferation of erythroid precursors $>80 \%$ of all nucleated cells, of which at least $30 \%$ are proerythroblasts ${ }^{11}$. MDS-erythroleukemia often refers to progression of disease from pre-existing MDS. In both PEL and MDSerythroleukemia, peripheral pancytopenia is common, with the marrow erythroblasts being medium-to-large in size, agranular and they exhibit deep basophilic cytoplasm. The neoplastic cells show reactivity with acid phosphatase, NSE and PAS cytochemistry. Erythroblasts usually express CD36 (early erythroid marker), glycophorin A/CD235a (late erythroid marker) and CD71 (transferrin receptor-1) but are negative for CD34 and HLA DR ${ }^{12}$. High frequency of TP53 mutations may indicate the importance of TP53 in the pathogenesis of the disease ${ }^{13}$. Presence of complex karyotype, AXL1, DNMT3a, 5q- and 7q- should prompt the possibility of MDS-erythroleukemia rather than de novo PEL. Prognosis of PEL and MDS-erythroleukemia is poor with a median survival of less than 6 months from diagnosis.

A combination chemotherapy regimen of daunorubicin, an anthracycline and cytarabine, an antimetabolite is used commonly as first line treatment in AMKL. However, the complete remission (CR) rate with this standard therapy is at $50 \%^{14}$. Those who achieve CR are only able to sustain as such for a brief period. Patients should be given the option of allogenic stem cell transplantation (Allo-SCT) at first remission but relapse rates post Allo-SCT are high. As of today, there are no novel agents to treat AMKL.

\section{Conclusion}

In summary, we report a rare case of transformation of myelodysplastic syndrome to AMKL in a middleaged gentleman who was refractory to induction and salvage chemotherapy. The risk factors for his poor outcome are advanced age, aggressive disease biology, underlying presence of MDS, poor response to induction chemotherapy, high lactate dehydrogenase and lack of good cytogenetic and molecular mutations. More intensive research into the molecular pathogenesis of this disease is required to achieve better therapeutic modalities as current conventional therapies are ineffective.

\section{Declarations}

\section{Author contributions}

G.K. analysed the data, designed the paper, and contributed to the writing of the manuscript. L.B.S made critical revisions and approved the final manuscript.

\section{Declaration of conflicting interests}

Both authors declare no potential conflicts of interests.

\section{Ethical approval}

Ethical approval is not required as this is not a clinical trial.

\section{Funding}

Self-funding.

Guarantor 
Ganesh Kasinathan is the guarantor of this manuscript.

Informed consent

Written informed consent was obtained from the patient for publication of this case report and any accompanying images. A copy of the written consent is available for review by the Editor-in-Chief of this journal.

Acknowledgements

None

References

1. Bennett JM, Catovsky D, Daniel MT, et al. Criteria for the diagnosis of acute leukemia of megakaryocyte lineage (M7). A report of the French-American-British Cooperative Group. Ann Intern Med. 1985; 103:460-462.

2. Gamis AS. Acute myeloid leukemia and Down syndrome evolution of modern therapy: state of the art review. Pediatr Blood Cancer. 2005; 44:13-20.

3. Mukherjee S, Ibrahimi S, John S, et al. Non-seminomatous mediastinal germ cell tumour and acute megakaryoblastic leukemia. Ann Hematol.2017; 96(9):1435-1439.

4. Pagano L, Pulsoni A, Vignetti M, et al. GIMEMA. Acute megakaryoblastic leukemia: experience of GIMEMA trials. Leukemia, 2002; 16(9):1622-1626.

5. Huang MJ, Li CY, Nicholas WL, et al. Acute leukemia with megakaryocytic differentiation: A study of 12 cases identified immunocytochemically. Blood. 1984; 64:427-439.

6. Masoumi-Dehshiri R, Hashemi AS, Neamatzadeh H, et al. A Case Report: Acute Myeloid Leukemia (FAB M7). Iran J Ped Hematol Oncol. 2014; 4(4):188-190.

7. Shibata A, Bennett JM, Castoldi GL, et al. Recommended methods for cytological procedures in hematology. International committee for standardization in hematology (ICSH). Clin Lab Haematol. $1985 ; 7: 55-74$.

8. Oki Y, Kantarjian HM, Zhou X, et al. Adult acute megakaryocytic leukemia: An analysis of 37 patients treated at M.D. Anderson Cancer Centre. Blood. 2006; 107:880-884.

9. Mazzella F, Alvares C, Kowal-Vern A, et al. The acute erythroleukemias. Clin Lab Med. 200; 20:119137.

10. Ahmad F, Dalvi R, Das BR, et al. Novel t(8;17)(q23;q24.2) and t $(9 ; 22)(\mathrm{p} 24.1 ; \mathrm{q} 12.2)$ in acute megakaryoblastic leukemia AML-M7 subtype in an adult patient. Cancer Genet Cytogenet.2009; 193:112-115.

11. Arber DA, Orazi A, Hasserjian R, et al. The 2016 revision to the World Health Organization (WHO) classification of myeloid neoplasms and acute leukemia. Blood. 2016; 127:2391-2405.

12. Hasserjian RP. Erythroleukemia and its differential diagnosis. Surg Pathol Clin. 2013; 6:641-659.

13. Wang SA, Hasserjian RP. Acute erythroleukemias, acute megakaryoblastic leukemias, and reactive mimics: a guide to a number of perplexing entities. Am J Clin Pathol. 2015;144:44-60.

14. Garderet L, Labopin M, Gorin NC, et al. Hematopoietic stem cell transplantation for de novo acute megakaryocytic leukemia in first remission: A retrospective study of the European Group for Blood and Marrow Transplantation (EBMT). Blood. 2005; 105:405-419.

Figure legends

\section{Figure 1}

Figure 1: (A) The bone marrow aspirate demonstrates hypercellularity for age, trilineage dysplasia with $52 \%$ megakaryoblasts. The megakaryoblasts are described as agranular, medium-to-large cells with round nucleus, scanty basophilic cytoplasm with cytoplasmic blebs and pseudopods. Erythropoiesis and granulopoiesis are suppressed. (B) H \& E stain of trephine biopsy (x 40 magnification) shows infiltration of marrow by large clusters of megakaryoblasts. (C-E) A panel of immunohistochemical staining, Dako, Denmark (x 40 magnifications) shows positivity for CD34 and CD61 and negativity for MPO. (F) Reticulin stain shows Grade 2 marrow fibrosis. 
Figure 1
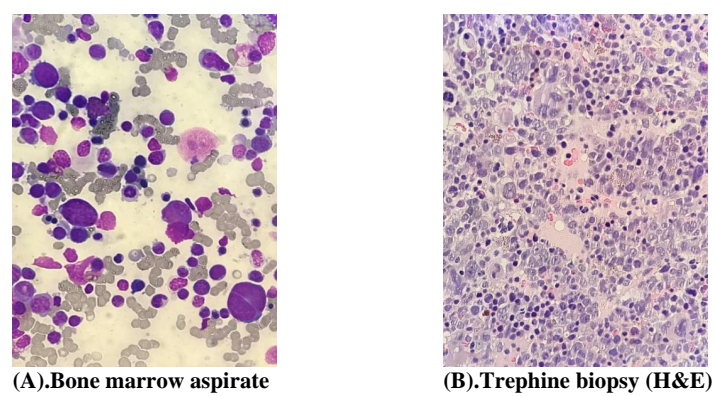

(B).Trephine biopsy (H\&E)
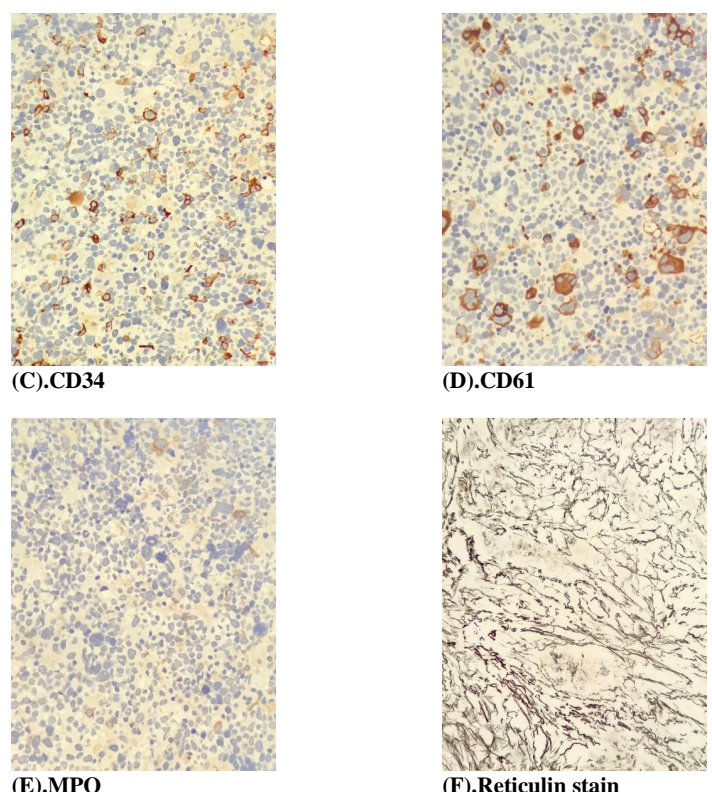